\title{
Lateral Oropharyngeal Wall Coverage with Buccinator Myomucosal and Buccal Fat Pad Flaps
}

\author{
Bok Ki Jung ${ }^{1}$, Seung Yong Song ${ }^{1}$, Se-Heon Kim², Young Seok Kim³ ${ }^{3}$, Won Jai Lee ${ }^{1}$, \\ Jong Won Hong ${ }^{1}$, Tai Suk Roh ${ }^{3}$, Dae Hyun Lew ${ }^{1}$ \\ Departments of ${ }^{1}$ Plastic and Reconstructive Surgery and ${ }^{2}$ Otorhinolaryngology, Institute for Human Tissue Restoration, Severance Hospital, \\ Yonsei University College of Medicine, Seoul; ${ }^{3}$ Department of Plastic and Reconstructive Surgery, Institute for Human Tissue Restoration, \\ Gangnam Severance Hospital, Yonsei University College of Medicine, Seoul, Korea
}

Background Reconstruction of oropharyngeal defects after resection of oropharyngeal cancer is a significant challenge. The purpose of this study is to introduce reconstruction using a combination of a buccinator myomucosal flap and a buccal fat pad flap after cancer excision and to discuss the associated anatomy, surgical procedure, and clinical applications.

Methods In our study, a combination of a buccinator myomucosal flap with a buccal fat pad flap was utilized for reconstruction after resection of oropharyngeal cancer, performed between 2013 and 2015. After oropharyngectomy, the defect with exposed vital structures was noted. A buccinator myomucosal flap was designed and elevated after an assessment of the flap pedicle. Without requiring an additional procedure, a buccal fat pad flap was easily harvested in the same field and gently pulled to obtain sufficient volume. The flaps were rotated and covered the defect. In addition, using cadaver dissections, we investigated the feasibility of transposing the flaps into the lateral oropharyngeal defect.

Results The reconstruction was performed in patients with squamous cell carcinoma. The largest tumor size was $5 \mathrm{~cm} \times 2 \mathrm{~cm}$ (length $\times$ width). All donor sites were closed primarily. The flaps were completely epithelialized after four weeks, and the patients were followed up for at least six months. There were no flap failures or postoperative wound complications. All patients were without dietary restrictions, and no patient had problems related to mouth opening, swallowing, or speech.

Conclusions A buccinator myomucosal flap with a buccal fat pad flap is a reliable and valuable option in the reconstruction of oropharyngeal defects after cancer resection for maintaining functionality.

Keywords Surgical flap / Oropharyngeal neoplasms / Reconstructive surgical procedures
Correspondence: Jong Won Hong

Department of Plastic and Reconstructive Surgery, Yonsei University College of Medicine, 50 Yonsei-ro, Seodaemun-gu, Seoul 120-752, Korea

Tel: $+82-2-2228-2215$

Fax: +82-2-393-6947

E-mail: hsaturn@hanmail.net
I would like to express my sincere gratitude to professor Hyung Woo, PARK and the staff of Surgical Anatomy Education Center of Yonsei university medical school for assisting this study.

No potential conflict of interest relevant to this article was reported.

\section{INTRODUCTION}

Recently, minimally invasive approaches such as transoral robotic surgery have been developed for the management of oro- pharyngeal cancers [1]. The parapharyngeal space (PPS) is a difficult area to access due to its anatomical complexity and relationship with the surrounding vital structures, such as the carotid artery, internal jugular vein, and cranial nerves IX to XII [1]. 
Pollei et al. [2] suggested that the exposed branches of the external carotid artery must be ligated in the resection bed and coverage should be achieved with local flaps or surrounding tissues, typically including the lingual and facial arteries in oropharyngeal resections.

Reconstruction of oropharyngeal defects after resection of oropharyngeal cancer is a significant challenge for the reconstructing surgeon in terms of ensuring favorable functional and esthetic outcomes. Oral cavity defects after tumor resection can be reconstructed depending on the size and anatomic position of the defects. Traditionally, the oral cavity defects resulting from tumor resection were reconstructed using skin grafts, locoregional flaps, and free flaps [3]. Ideal reconstruction should be accomplished by matching the resected tissue in texture, color, consistency, bulk, and minimal donor site morbidity. In addition, the flap should be easily harvested and versatile [4].

Many surgeons have described the use of intraoral local flaps from the oral mucosa. Currently, they use a buccinator myomucosal flap [5] based on the buccal artery, and a facial artery and a buccal fat pad flap [6] for the reconstruction of oropharyngeal defects. However, to date, there have been no published data on the reconstruction for lateral oropharyngeal defects by using two flaps, namely a buccinator myomucosal flap and a buccal fat pad flap. Therefore, the purpose of this study was to introduce our reconstructive technique and novel use of a combination of the buccinator myomucosal flap with a buccal fat pad flap to cover defects of the lateral oropharyngeal wall after cancer excision and to discuss the associated anatomy, surgical procedure, and clinical applications. In addition, we present the anatomic and cadaveric foundations for the buccinator myomucosal flap and the buccal fat pad flap for reconstruction of the lateral oropharyngeal wall.

\section{METHODS}

\section{Patients}

In our study, reconstructions with buccinator myomucosal and buccal fat pad flaps after transoral robotic oropharyngeal cancer resection were performed between 2013 and 2015. Patient bio- logic information, pathology, cancer stage, reconstructive methods, and adjuvant therapy were recorded (Table 1).

One fresh and two preserved human specimens were used for anatomic dissections. Utilizing anatomic and surgical dissections, we investigated the feasibility of transposing the buccinator myomucosal flap and the buccal fat pad flap into the lateral oropharyngeal cavity. Both flaps were raised, and techniques for transposition into the lateral oropharyngeal cavity were investigated.

\section{Surgical techniques}

After transoral robot-assisted oropharyngectomy was performed by the otorhinolaryngology team, the branch of the external carotid artery was exposed. The orifice of stensen's duct in the cheek was identified and tagged with a suture. The buccinator myomucosal flap was designed on the oral mucosa, and the facial artery was outlined using Doppler. The designed flap measured up to $4 \mathrm{~cm} \times 2 \mathrm{~cm}$ (width $\times$ length) [7]. The flap must be placed below Stenson's duct and at least $1.5 \mathrm{~cm}$ away from the oral commissure. Lidocaine (1\%) mixed with 1:100,000 epinephrine was infiltrated into the operative field except for in the vicinity of the pedicle. With respect to the approach below the orifice of Stenson's duct through the medial wall of the buccal space, the incision started from the distal end of the flap through the mucosa and the buccinator muscle while preserving the pedicles. Skeletonization of the pedicle was not completely performed. The dissection proceeded deep and posteriorly, taking the overlying buccinator and orbicularis oris muscles. The harvested buccinator myomucosal flap, which is based on the facial artery inferiorly, is then transposed onto the defect by simply rotating it around a pivot at the base of the vascular pedicle to avoid kinking or twisting the vessels.

Elevation of the buccal fat pad flap did not require an additional incision or extended dissection. The buccal fat pad flap was elevated under the buccinator myomucosal flap through the second molar area. External digital pressure was placed on the cheek to facilitate herniation of the buccal fat pad. The buccal fat pad became immediately visible and was gently pulled to obtain sufficient length. The buccal fat pad should be free and easi-

\begin{tabular}{|c|c|c|c|c|c|c|c|c|c|}
\hline No. & $\begin{array}{l}\text { Age } \\
\text { (yr) }\end{array}$ & Sex & $\begin{array}{l}\text { Tumor } \\
\text { site }\end{array}$ & $\begin{array}{l}\text { Pathologic } \\
\text { diagnosis }\end{array}$ & Stage & $\begin{array}{c}\text { Size of } \\
\text { defect } \\
(\mathrm{cm} \times \mathrm{cm})\end{array}$ & $\begin{array}{l}\text { Buccinator } \\
\text { myomucosal } \\
\text { flap size } \\
(\mathrm{cm} \times \mathrm{cm})\end{array}$ & $\begin{array}{l}\text { Buccal fat pad } \\
\text { flap size } \\
(\mathrm{cm} \times \mathrm{cm})\end{array}$ & Complication \\
\hline 1 & 32 & Female & Tonsil & Squamous cell carcinoma & T2N2 & $4.0 \times 1.5$ & $5.0 \times 2.0$ & $5.0 \times 2.0$ & No \\
\hline 2 & 81 & Male & Tonsil & Squamous cell carcinoma & T2N2 & $4.0 \times 2.0$ & $5.0 \times 2.0$ & $5.0 \times 2.0$ & No \\
\hline 3 & 65 & Male & Tonsil & Squamous cell carcinoma & T4N2 & $6.0 \times 4.0$ & $5.0 \times 2.0$ & $6.0 \times 2.0$ & No \\
\hline
\end{tabular}


ly movable to avoid stretching the nerve structures.

The buccal fat pad flap was rotated and covered the oropharyngeal defect; then, the buccinator myomucosal flap was transposed onto the oropharyngeal defect without tension and fixed to the mucosal margin with 3-0 or 4-0 polyglycolic sutures. The donor defect was primarily closed or left uncovered.

\section{RESULTS}

All flaps were harvested and transposed successfully. The largest oropharyngeal defect measured $6 \mathrm{~cm} \times 4 \mathrm{~cm}$ (length $\times$ width). The largest buccinator myomucosal flap measured $5 \mathrm{~cm} \times 2 \mathrm{~cm}$ (length $\times$ width), and the length of the flap pedicle was $4.5 \mathrm{~cm}$. The largest buccal fat pad flap measured $6 \mathrm{~cm} \times 2 \mathrm{~cm}$ (length $\times$ width). The flap was rotated about $180^{\circ}$ inferiorly or superiorly and then fixed without any tension. The width of the donor site was as large as $2 \mathrm{~cm}$, so the site was closed primarily.

The flaps were completely epithelialized after four weeks. The patients were followed for at least six months. There were no flap failures or postoperative wound complications. All patients

Fig. 1. Case 1

A 32-year-old woman presented squamous cell carcinoma (T2N2M0) of the left tonsil: (A, B) a oropharyngeal defect after lateral oropharyngectomy, (C) flap design, (D) elevation of the flap, and $(E, F)$ immediate postoperative view.
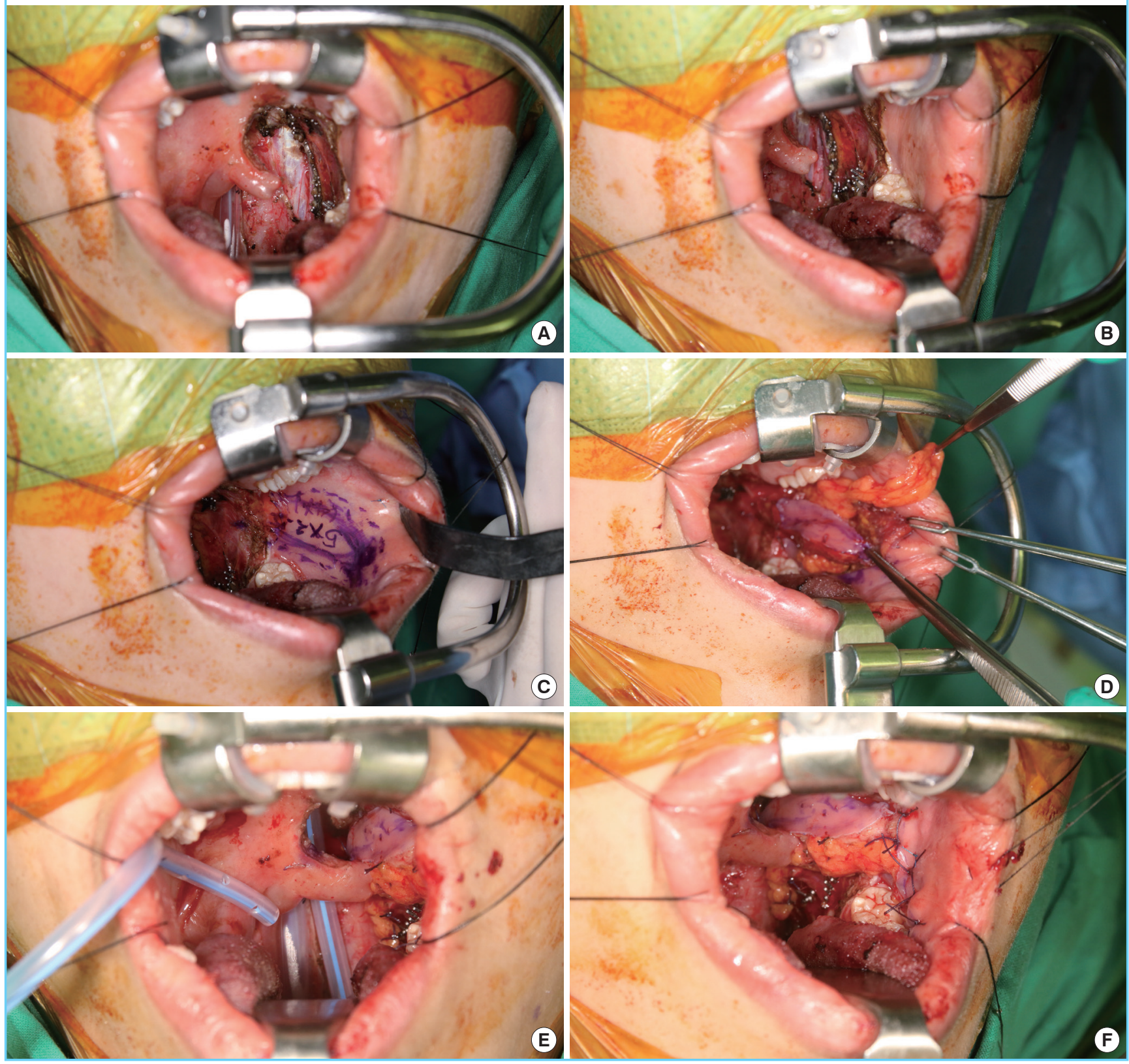
tolerated a normal diet, and no patient had problems related to mouth opening, swallowing, or speech.

\section{Case 1}

The patient was a 32-year-old woman with squamous cell carcinoma (T2N2M0) of the left tonsil. The patient underwent lateral oropharyngectomy and modified radical neck dissection to preserve the facial artery. The oropharyngeal defect measured about $4 \mathrm{~cm} \times 1.5 \mathrm{~cm}$, and the carotid artery was exposed. The oropharyngeal defect was reconstructed using a combination of a buccinator flap and a buccal fat pad flap. The size of the buccinator myomucosal flap measured about $5 \mathrm{~cm} \times 2 \mathrm{~cm}$ as did the buccal fat pad flap. The flap was transposed inferiorly and covered the defect. The donor site was primarily closed (Fig. 1). The flap was completely re-epithelialized after four weeks. No infection or flap resorption was observed after ten months of follow-up (Fig. 2). She had no limitations with respect to mouth opening or trismus. The aesthetic results were good without cheek depression.

\section{Case 2}

The patient was an 81-year-old man with squamous cell carcinoma (T2N2M0) of the left tonsillar fossa. The patient underwent lateral oropharyngectomy and modified radical neck dissection to preserve the facial artery, accessory nerve, and internal jugular vein. The oropharyngeal defect measured about 4 $\mathrm{cm} \times 2 \mathrm{~cm}$, and the tortuous internal carotid artery was exposed. The oropharyngeal defect was reconstructed using a combination of the buccinator myomucosal flap and the buccal fat pad flap. The buccinator myomucosal and buccal fat pad flaps were each about $5 \mathrm{~cm} \times 2 \mathrm{~cm}$. The flap was transposed inferiorly and covered the defect. Some of the donor site was closed, and the remaining area was left open with the expectation of secondary healing.

The flap and the donor site were completely re-epithelialized after four weeks. No wound complications such as infection or resorption of the buccal fat pad were observed after nine months of follow-up. He had no limitations in oral function (e.g., mouth opening or trismus). The aesthetic results were good without visible scars.

\section{Case 3}

The patient was a 65-year-old man with squamous cell carcinoma (T4N2M0) of the right palatine tonsil. As the tumor was adjacent to the internal carotid artery, he underwent neo-adjuvant chemotherapy for 16 weeks. He subsequently underwent lateral oropharyngectomy and modified radical neck dissection to preserve the facial artery. The oropharyngeal defect measured about $6 \mathrm{~cm} \times 4 \mathrm{~cm}$, and the internal carotid artery was exposed. The oropharyngeal defect was reconstructed using a combination of the buccinator myomucosal flap with the buccal fat pad flap. The buccinator myomucosal flap measured about $5 \mathrm{~cm} \times 2$ $\mathrm{cm}$, and the buccal fat pad flap measured about $6 \mathrm{~cm} \times 2 \mathrm{~cm}$. The flap was transposed inferiorly and covered the defect. Some of the donor site was closed, and the remaining area was left open with the expectation of secondary healing.

A minor wound complication occurred, and a revision was done. The flap and the donor site were completely re-epithelialized after four weeks. There were no wound complications including infection or resorption of the buccal fat pad after six months of follow-up. The patient was able to open his mouth without difficulty and had no trismus.

\section{Fig. 2. Postoperative intraoral view}

The entire surface of the flap was completely re-epithelialized, and no infection or flap resorption was observed after ten months of follow-up.
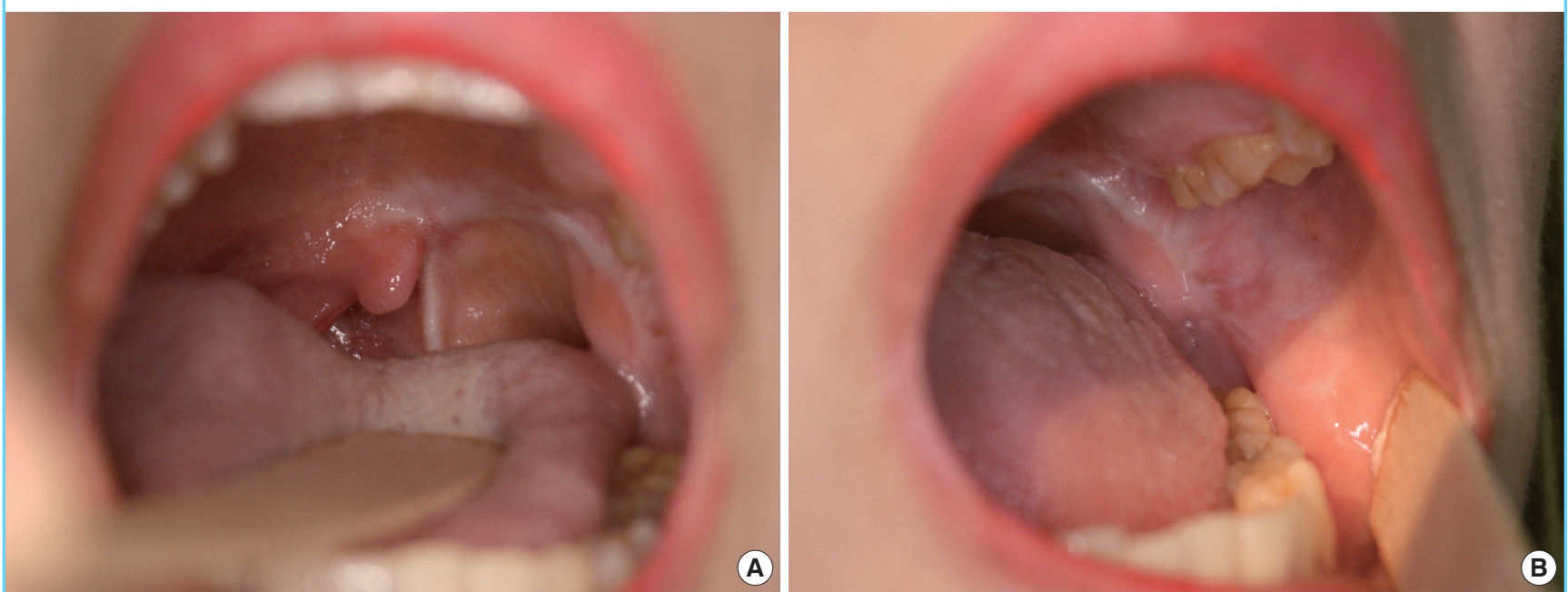


\section{Fig. 3. Anatomy of facial vessels and buccal fat pad}

(A) Facial artery and vein, and (B) buccal fat pad is sufficiently large to cover the lateral oropharyngeal cavity.
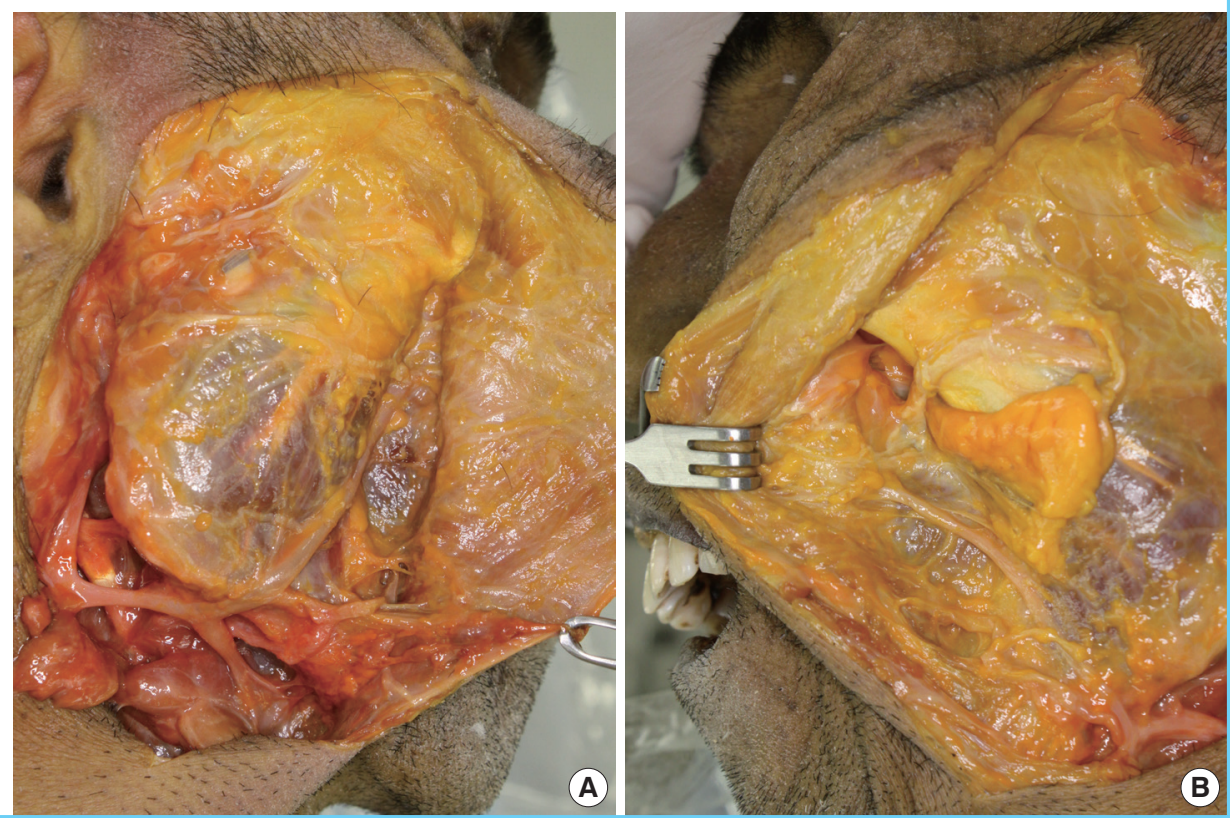

\section{Cadaveric study}

The anatomy of the buccinators, buccal fat pad, and facial vessels has been extensively described. In brief, the facial artery branches from the external carotid artery and enters the face by crossing the inferior border of the mandible and ascending toward the nasal alae. The facial artery supplies the buccinator muscle through inferior and anterior buccal branches and ascends in the nasofacial groove to the medial canthus as the angular artery (Fig. 3A). The anastomoses among arteries supplying the buccinators muscle could not be found in our cadaver, as was in our cadaver. The facial artery and vein were in close proximity to the angle of the mandible. The buccal fat pad flap were encapsulated and distinct from the subcutaneous fat. Its dissection was performed by mobilization of its body and buccal process on a vascularized pedicle (Fig. 3B).

Through the intraoral incision (Fig. 4A), the buccinator myomucosal flap was elevated in the cadaver without injury to the orifice of the parotid duct. The pedicle of the facial artery was not skeletonized or included in the flap because of the preservation of the blood supply network (Fig. 4B). The buccal fat pad flap was then elevated in the same operative field (Fig. 4C). The flap was long enough to translocate the buccal fat pad flap to the lateral oropharyngeal defect (Fig. 4D, E).

\section{DISCUSSION}

Several methods for oropharyngeal reconstruction secondary to cancer resection have been described. The choice of treatment depends on many factors such as the size of the defect, the position of the defect, the volume or condition of surrounding tissue, functional restoration, and aesthetic results. No reconstruction is needed for small defects because they heal spontaneously or can be closed primarily.

Several prior studies described skin grafts and locoregional flaps for reconstruction of moderately sized defects of the oral cavity [8-12]. Although a split thickness skin graft is simple to harvest, is thin, and can be applied to a large defect, it can result in contractures that interfere with the restoration of normal oral functions such as mouth opening and cannot be used to cover exposed vital structures such as bone and vessels [13]. Vital structures such as carotid artery, jugular vein, and cranial nerve are often exposed after oropharyngectomy. In a situation where the exposed structure is not completely covered, postoperative bleeding can occur. Pollei et al. [2] reported that postoperative bleeding occurred in $5.4 \%$ of the patients with $67.3 \%$ of them requiring operative intervention, and severe life threatening bleeding occurred in $1.1 \%$ of the patients. Therefore, adequate coverage of critical structures is a key component of defect reconstruction.

The disadvantages of locoregional flaps include the limitation in size of the flap supplied with a certain pedicle and postoperative contractures, which can result in limited mouth opening, trismus, and donor site morbidity. Reconstruction using a locoregional flap is not appropriate for moderate-to-large defects. Defects, too large for primary repair, can be reconstructed using free flaps. Free flaps are the first choice for complex intraoral reconstructions involving the tongue, floor of the mouth, palate, 


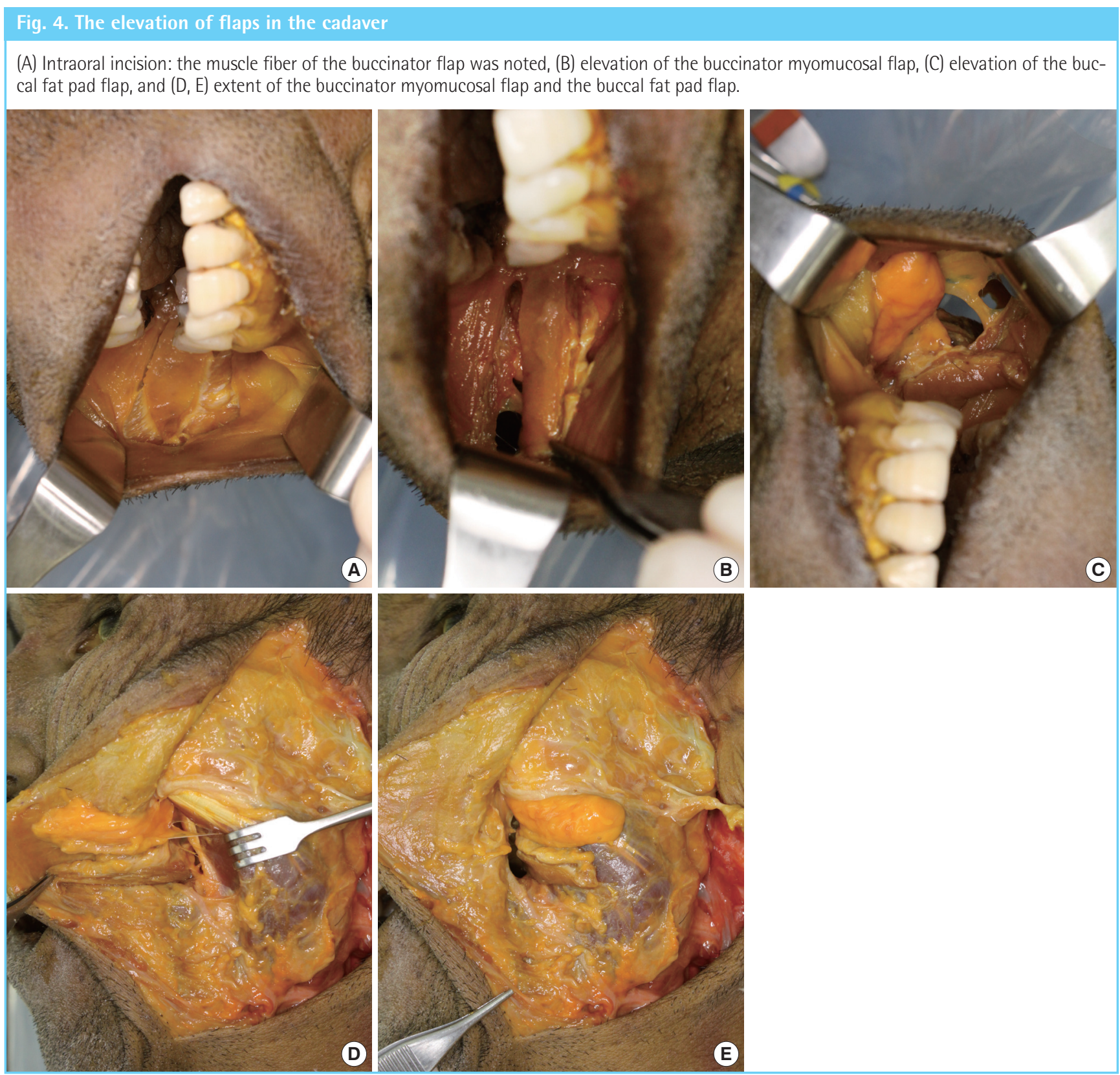

cheek mucosa, and lip $[14,15]$. Although free flaps are the best choice for large defects, there are several disadvantages in terms of flap bulkiness, donor site morbidity, and long and complex surgery. The ideal reconstruction should completely cover the defects and replace the original tissue with the same or similar type of tissue. The reconstructed tissue should be similar to the native tissue in terms of mucosal sensitivity, volume, and texture [16].

The buccinator myomucosal flap and the buccal fat pad flap are suitable options for covering a buccal defect. The buccinator muscle is a thin, rectangular-shaped cheek muscle that is involved in actions such as whistling, sucking, and mastication [4]. At its medial aspect, the buccinator muscle is covered with the mu- cosa and submucosa of the cheek, and at its lateral aspect, it contacts the mandible, masseter muscles, buccal fat pad, and buccopharyngeal fascia [3]. The buccinator muscle has a rich blood supply from the network between the facial and the buccal arteries [17]. Zhao et al. [18] reported that a buccinator myomucosal flap can be harvested as a pedicled flap or axial flap based on the buccal or facial artery and can be based anteriorly, posteriorly, or superiorly. The posteriorly based flap is useful for the coverage of the tonsil bed, soft and hard palate, and retromolar trigone, while the anteriorly based flap is good for the reconstruction of the lip, floor of the mouth, and tongue. The superiorly based flap has been described in the repair of defects in the anterior hard palate, alveolus, and lips, as well as the orbit. However, 
this flap is limited by the size and volume needed to cover the defect.

The buccinator myomucosal flap has several advantages. It has great versatility for reconstructing small-to-moderately sized intraoral defects, can be flexibly designed in various shapes, and has minimal donor site morbidity with minimal scarring due to the possibility of closure with sutures. Most importantly, it is similar to the original tissues in terms of texture, color, and sensitivity. The buccinator flaps maintain mucous secretion and sensitivity and oral functions such as speaking and swallowing [16]. Harvesting the flap is technically easy as compared to free flap harvest, so the operating time is shorter than that of reconstruction using free flaps or local flaps.

Egyedi [19] suggested the use of a buccal fat pad flap for intraoral reconstruction. The buccal fat pad is an encapsulated, complex, three-dimensional structure with neurovascular bundles, distinct from the subcutaneous fat [20]. It lies between the buccinator muscle and the masseter muscle [3]. The flap can be applied to reconstruct defects of the hard palate, soft palate, buccal mucosa, and posterior maxilla [21]. It has a rich blood system sufficient for the coverage of a small-to-moderately sized oral defect. In addition, the buccal fat pad flap is flexible to design, is simple to access, has low morbidity, and has excellent re-epithelization [22].

Few studies have described the combination of a buccinator myomucosal flap with a buccal fat pad flap [22]. In our experience, the use of a buccinator flap alone was limited in terms of the length and volume of the flap for defect coverage. Furthermore, while the buccal fat pad flap was sufficient with respect to size or volume, the fat tissue was vulnerable to tension or external forces. Thus, we used the buccinator flap with the buccal fat pad flap for the coverage of oropharyngeal defects in this study.

In our cases, after tumor removal, the largest oropharyngeal defect noted measured $6 \mathrm{~cm} \times 4 \mathrm{~cm}$ with the internal carotid artery exposed. As previously stated, the mean pedicle length of buccinator myomucosal flaps ranges from 4.2 to $5.5 \mathrm{~cm}$, and the paddle size of the flap ranges from $2.2 \mathrm{~cm} \times 2.1 \mathrm{~cm}$ to $3.5 \mathrm{~cm} \times 3$ $\mathrm{cm}$ [7]. In order to obtain a sufficient flap volume and length, we used the combination of a buccinator myomucosal flap with a buccal fat pad flap. This approach provides additional available tissue volume for defect coverage, allowing larger defects to be reconstructed than with the use of any flap alone.

In the process of flap design, the width of the flap was not to exceed $2.5 \mathrm{~cm}$ for primary closure. The opening of Stenson's duct was preoperatively marked with a suture to avoid damaging it.

Incision and dissection are both needed when using a buccinator flap or a buccal fat pad flap. When using a buccinator myo- mucosal flap and a buccal fat pad flap together, the operative field is the same. After elevation of the buccinator flap, only simple dissection is needed to elevate the buccal fat pad flap from the exposed raw surface. The use of these flaps for the same reconstruction has several advantages including an easy, relatively fast surgery and intraoral harvest.

During elevation of the flap, we were focused on the dissection of the pedicle to preserve blood supply to the entire flap. The artery and vein surrounding the buccinator muscle organized the complicated blood network. The buccinator muscle has a rich blood system from the anastomosis between the facial artery and the external carotid artery, the posterior buccal branch of the facial artery, the internal maxillary artery, the buccal artery, and the posterosuperior alveolar artery. In addition, the vein drains into the buccal venous plexus, the pterygoid plexus, the internal maxillary vein, and the deep facial vein [18]. During elevation of the buccinator flap, the pedicle was not clearly identified and the vascular pedicle should be just wide enough to be rotated on a pivot point in order to maintain the blood supply network because the blood supply and the venous drainage of the flap come from the network of small arterioles and venules.

The blood supply to the buccal fat pad flap is mostly through a network of vessels surrounding the capsule. Therefore, the surgeon must take care to keep the capsule and vessels intact during dissection of the flap. Subsequently, a part of the capsule is separated to obtain sufficient length in a range that does not interfere with the blood supply. The flap was rotated about $180^{\circ}$ to cover the defect. The direction of the rotation was either superiorly rotated or inferiorly to avoid kinking of the pedicle and tension.

Because of the tiny diameters of vessels in the cadaveric study, there were no networks of blood vessels found around the buccinator flap and the buccal fat pad flap. However, it is certain that not skeletonizing the pedicle of the flap and preserving the network of vessels are helpful for the transposition of the buccinator flap and the buccal fat pad flap. In two different specimens, we found that the lengths and volumes of the buccinator flap and the buccal fat pad flap are sufficient to cover the lateral oropharyngeal defect.

Dissecting the buccal area always involves the risk of facial nerve trunk damage. However, when we checked the location and depth of the flap in the cadaver, damages to the digital buccal branches seemed possible, while direct damages to the trunk itself seemed very unlikely.

Our study has several limitations. The main limitation is the small number of the patients who underwent oropharyngeal reconstruction using the buccinator flap with the buccal fat pad flap. While other studies utilized a skeletonized pedicle in defect 
reconstruction, in our study, we determined that the pedicle was wide enough to maintain appropriate blood supply, and no complications occurred. Further studies with an anatomic and cadaveric foundation should be done to characterize the blood supply system. A compilation of more cases and cadaver specimens will aid a deeper understanding of the buccinator and buccal fat pad flaps.

\section{REFERENCES}

1. Park YM, Lee JG, Lee WS, et al. Feasibility of transoral lateral oropharyngectomy using a robotic surgical system for tonsillar cancer. Oral Oncol 2009;45:e62-6.

2. Pollei TR, Hinni ML, Moore EJ, et al. Analysis of postoperative bleeding and risk factors in transoral surgery of the oropharynx. JAMA Otolaryngol Head Neck Surg 2013;139: 1212-8.

3. Squaquara R, Kim Evans KF, Spanio di Spilimbergo S, et al. Intraoral reconstruction using local and regional flaps. Semin Plast Surg 2010;24:198-211.

4. Szeto C, Yoo J, Busato GM, et al. The buccinator flap: a review of current clinical applications. Curr Opin Otolaryngol Head Neck Surg 2011;19:257-62.

5. Van Lierop AC, Fagan JJ. Buccinator myomucosal flap: clinical results and review of anatomy, surgical technique and applications. J Laryngol Otol 2008;122:181-7.

6. Kim JT, Naidu S, Kim YH. The buccal fat: a convenient and effective autologous option to prevent Frey syndrome and for facial contouring following parotidectomy. Plast Reconstr Surg 2010;125:1706-9.

7. Rahpeyma A, Khajehahmadi S, Rezvani HN. Pedicle length in the inferiorly based buccinator myomucosal island flap: a cadaveric study. Int J Oral Maxillofac Surg 2014;43:173-6.

8. Bande CR, Datarkar A, Khare N. Extended nasolabial flap compared with the platysma myocutaneous muscle flap for reconstruction of intraoral defects after release of oral submucous fibrosis: a comparative study. $\mathrm{Br} \mathrm{J}$ Oral Maxillofac Surg 2013;51:37-40.

9. Rahpeyma A, Khajehahmadi S. Submental artery island flap in intraoral reconstruction: a review. J Craniomaxillofac Surg 2014;42:983-9.

10. Michaelidis IG, Hatzistefanou IM. Functional and aesthetic reconstruction of extensive oral ablative defects using temporalis muscle flap: a case report and a sort review. J Craniomaxillofac Surg 2011;39:200-5.

11. Yin XM, Li Y, Lu XZ, et al. Pectoralis major myocutaneous flap for repairing large tissue defects following oral cancer surgery. Nan Fang Yi Ke Da Xue Xue Bao 2010;30:2546-7.

12. Lee DW, Yun IS, Rah DK, et al. Effectiveness of the trapezius vein in the reconstruction of intraoral defects with bare neck. J Craniofac Surg 2010;21:1423-7.

13. Rhee PH, Friedman CD, Ridge JA, et al. The use of processed allograft dermal matrix for intraoral resurfacing: an alternative to split-thickness skin grafts. Arch Otolaryngol Head Neck Surg 1998;124:1201-4.

14. Cymerman JA, Kulkarni R, Gouldesbrough D, et al. First report of squamous cell carcinoma arising within an intraoral radial forearm free flap. Int J Surg Case Rep 2013;4:731-4.

15. Sun G, Lu M, Hu Q, et al. Clinical application of thin anterolateral thigh flap in the reconstruction of intraoral defects. Oral Surg Oral Med Oral Pathol Oral Radiol 2013;115:18591.

16. Massarelli O, Baj A, Gobbi R, et al. Cheek mucosa: a versatile donor site of myomucosal flaps: technical and functional considerations. Head Neck 2013;35:109-17.

17. Ferrari S, Ferri A, Bianchi B, et al. A novel technique for cheek mucosa defect reconstruction using a pedicled buccal fat pad and buccinator myomucosal island flap. Oral Oncol 2009; 45:59-62.

18. Zhao Z, Li S, Yan Y, et al. New buccinator myomucosal island flap: anatomic study and clinical application. Plast Reconstr Surg 1999;104:55-64.

19. Egyedi P. Utilization of the buccal fat pad for closure of oroantral and/or oro-nasal communications. J Maxillofac Surg 1977;5:241-4.

20. Jackson IT. Anatomy of the buccal fat pad and its clinical significance. Plast Reconstr Surg 1999;103:2059-60.

21. Toshihiro Y, Nariai Y, Takamura Y, et al. Applicability of buccal fat pad grafting for oral reconstruction. Int J Oral Maxillofac Surg 2013;42:604-10.

22. Candamourty R, Jain MK, Sankar K, et al. Double-layered closure of oroantral fistula using buccal fat pad and buccal advancement flap. J Nat Sci Biol Med 2012;3:203-5. 